\title{
2 Wissenschaftliche Methodik und der Mehrwert einer Metaebene in der Wissenschaft
}

The wrong view of science betrays itself in the craving to be right; for it is not his possession of knowledge, of irrefutable truth, that makes the man of science, but his persistent and recklessly critical quest for truth.

Popper (1959/2002: 281)

\subsection{Das wissenschaftliche Programm}

Wissenschaft gehört sicherlich zu jenen Begriffen, zu welchen auch der wissenschaftliche Laie eine Meinung zu äußern vermag. So könnte eine derartige Erklärung des Konzeptes postulieren, Wissenschaft sei der Überbegriff zu verschiedenen theoretischen und praktischen Arbeitsfeldern wie der Medizin, der Raumfahrt und der Psychologie. Alternative Beschreibungen könnten sich darauf konzentrieren, wie die Wissenschaft die Gesamtheit menschlicher Erkenntnisse zur materiellen Welt sowie unserer eigenen gedanklichen Wirklichkeit umfasst oder wie die Wissenschaft die Systematik objektiver Untersuchung darstellt. Freilich ist keiner dieser Aspekte völlig abwegig, denn im Rahmen der Wissenschaft finden sich in der Tat eine Reihe von Disziplinen, innerhalb welcher Forscher beständig die Fixierung neuer Erkenntnisse verfolgen und dafür Qualitätskriterien wie eine möglichst objektive Gültigkeit heranziehen. Zugleich offenbart eine scheinbar naive, aus philosophischer Neugierde geborene Nachfrage, was denn jenseits jener vagen Beschreibungen die Wissenschaft im Kern ausmache, die Begrenztheit dieses laienhaften Wissenschaftsverständnisses.

Tatsächlich ist Wissenschaft weder ein Sammelbegriff für wissenschaftliche Disziplinen noch der Überbegriff für die Summe menschlichen Wissens - und auch wenn qualitativ hochwertige Wissenschaft mit Objektivität und weiteren Qualitätskriterien korreliert, so ist sie nicht mittels dieser Kriterien zu definieren. Poppers obiger Ausspruch beschreibt pointiert und mit großer Klarheit, dass Wissenschaftler nicht über das Volumen ihres deklarierten Wissens zu definieren sind, sondern über ihr hartnäckiges und rücksichtslos kritisches Streben nach Wahrheit. In ebendiesem Sinne ist es der Prozess der fortwährenden und stringenten Wissensschaffung, welcher die Wissenschaft definiert. In anderen, weniger redundanten Worten - d.h., sodass Wissenschaft nicht schlicht zu Wissensschaffung umformuliert wird - ist die Wissenschaft über ihre Methoden zu definieren: Ein sich beständig erweiterndes und verfeinerndes System aus Vorgehensweisen, welches mit größtmöglicher Reliabilität zu wahr- 
heitsgetreuen Ergebnissen zu führen sucht. Dieses wissenschaftliche Programm geschieht im Kontext eines Konglomerats mehrerer grundlegender Prinzipien, vieler Arbeitsweisen bzw. Studiendesigns sowie unzähliger konkreter Methoden respektive Versuchsprotokollen und ist niemals als ein vollständig abgeschlossener Prozess zu sehen, sondern stattdessen als eine sukzessive, asymptotische Annäherung zur Wahrheit.

Im Folgenden sollen in knappster Weise mehrere grundlegende Begrifflichkeiten und Vorgehensweisen der Wissenschaft eingeführt und beschrieben werden, um zwischen dem Leser und der vorliegenden Arbeit eine operationalisierte, gemeinsame Terminologie zu schaffen. Auf dieser Basis wird der wissenschaftliche Prozess bis zur Ebene von Metastudien und systematischen Übersichtsarbeiten zu skizzieren sein, denn ein derartiges Verständnis schafft die Voraussetzung für das methodische Programm der vorliegenden Arbeit. Hierzu ist als eine erste Anmerkung festzustellen, dass das interdisziplinäre Forschungsfeld des Ursprungs menschlicher Sprachfähigkeit trotz der Indirektheit der triangulierenden Forschungstätigkeiten ein weitestgehend empirisches Unterfangen darstellt. Dies fußt erstens darin, dass die an dieser Triangulation beteiligten Einzelwissenschaften weitgehend empirisch vorgehen, und gilt zusätzlich, da auch die einschlägige Theoriebildung zum Ziel hat, eine in letzter Konsequenz deskriptive Beschreibung zum Zustandekommen des Untersuchungsgegenstands der menschlichen Sprachbefähigung zu generieren - d.h. sie hat einen empirischen Zielbereich. Die folgende Einführung beschäftigt sich in diesem Sinn mit empirisch orientierten wissenschaftlichen Methoden und operationalisiert die Terminologie in ebendiesem Sinn.

\subsection{Verifikation, Falsifikation und Vorhersagbarkeit}

Prinzipiell lassen sich wissenschaftliche Methoden so einordnen, dass sie drei generellen Prinzipien folgen. $\mathrm{Zu}$ Beginn steht das historisch älteste und archaische Prinzip der Verifikation. Eine Behauptung zu belegen, indem gezeigt wird, dass ihr Inhalt der Realität entspricht, erscheint als äußerst eingängig. Jedoch hat ein derartiges Vorgehen durchaus eng gesteckte Grenzen der Anwendbarkeit. Ein großer Teil zu belegender Behauptungen besteht nicht in simplen Existenzaussagen, für die eine Verifikation gemeinhin ausreicht, sondern sucht sich in weiter reichenden Aussagen wie Naturgesetzen, kategorischen Aussagen oder sonstigen Allsätzen. Wie bereits die frühneuzeitliche Philosophiegeschichte feststellte, sind derartige allgemeingültige Feststellungen jedoch prinzipiell nicht abschließend über das Prinzip der Verifikation zu belegen - eine Beweisbarkeit bleibt allein den deduktiven Logiken vorbehalten. Ein Einwand gegen 
diese Äußerungen besteht sicherlich darin, dass in einer pragmatischen Herangehensweise eine hinreichende induktive Annäherung an einen Nachweis durch das Prinzip der Verifikation durchaus möglich ist. Allerdings handelt es sich dabei, wenn mit nennenswerter Sorgfalt vorgegangen werden soll, um eine zeit- sowie ressourcenaufwendige und damit für das wissenschaftliche Programm gegebenenfalls hinderliche Vorgehensweise.

Stattdessen fußt die jüngere Forschungsgeschichte hinsichtlich einer Bewertung der Validität der verschiedenen Typen von Allsätzen auf dem Prinzip der Falsifikation. ${ }^{2}$ In diesem Sinne gilt die Untersuchung einer Hypothese nicht dem Versuch, sie $\mathrm{zu}$ belegen, sondern stattdessen, sie $\mathrm{zu}$ widerlegen. Hält ebendiese Hypothese einem solchen Prozess stand, so wird sie hierdurch mehr gestützt als durch ein Belegen mittels des gemeinhin selektiven und damit fehleranfälligen Ansatzes der Verifikation. Aus dieser Beschreibung ist freilich nicht zu folgern, dass einem positiven Nachweis kein wissenschaftlicher Mehrwert zu entnehmen ist, sondern lediglich, dass es dem zusätzlichen und mitunter effektiveren Prinzip der Falsifikation bedarf, wenn mit großer wissenschaftlicher Potenz gearbeitet werden soll. In letzter Konsequenz ist auch durch das Prinzip der Falsifikation jenseits von Existenzaussagen hinsichtlich empirischer Fragestellungen kein Beweis möglich, jedoch kann eine trotz ausgiebiger Untersuchung nicht falsifizierte Hypothese als provisorisch wahr angenommen werden - und damit besteht ein nennenswerter Mehrwert gegenüber einer reinen Verifikation ebendieser Hypothese.

Das dritte Prinzip, welches aktuell quasi den Goldstandard der wissenschaftlichen Validität von Hypothesen und insbesondere Theorien definiert, liegt in der produktiven Vorhersagbarkeit. Gemeint sei damit freilich nicht die inhärent immer gegebene Vorhersage einer Hypothese im Sinne ihres eigenen Inhalts - so beispielsweise die Vorhersage einer Hypothese im Sinne der Gravitationstheorie, dass ein Stift, wenn fallengelassen, nach „unten“ fällt. Vielmehr stellt eine derartige Hypothese selbst eine Ableitung dar und entspricht damit einer produktiven Vorhersage der Gravitationstheorie. Dies ist der Fall, da die Aussage, dass Dinge, die angehoben und losgelassen werden, nach unten fallen, nicht der zentralen Erkenntnis der Gravitationstheorie entspricht. Ganz im Gegensatz handelt es sich bei dieser Aussage um eine abgeleitete Hypothese mit

2 Dem Prinzip der Falsifikation folgt nicht nur der nachfolgend beschriebene Mehrwert, sondern auch der prinzipielle Ausschluss von nicht falsifizierbaren Erklärungsmodellen - eine Vorgehensweise, welche dem wissenschaftlichen Prozess nicht nur dienlich ist, sondern darüber hinaus eine Notwendigkeit darstellt, wenn derselbe leistungsfähig und produktiv verbleiben soll. 
darauf folgender und wiederholbarer empirischer Bestätigung. Die hintergründige Theorie hat stattdessen die tatsächliche physikalische Krümmung des Raumes bei einem Vorhandensein von Masse zum Gegenstand und erklärt neben jener bestätigten Hypothese auch noch weitere Phänomene. Sie vermag zu ergründen, weshalb Flüsse in letzter Konsequenz im Ozean enden und auf welche Weise die Aquädukte des antiken Roms ihr Wasser über weite Strecken transportieren konnten, aus welchem Grund versierte Pfeilschützen nicht auf ihr direktes Ziel ansetzen, sondern dem Flug ihres Pfeils eine flache Parabel unterstellen, warum die Erde sowie weitere hinreichend massereiche Himmelskörpern näherungsweise eine Kugelform angenommen haben und ebenso, wie der Mond auf seiner Umlaufbahn um die Erde sowie die Erde auf der ihrigen um die Sonne verbleibt. Alle diese und weitere Dinge haben einen gemeinsamen Erklärungshintergrund: Die moderne Gravitationstheorie, deren zentraler Mechanismus wie angemerkt in der Krümmung der Raumzeit unter dem Vorhandensein von Masse besteht.

In diesem Sinne stellt diese wie eine jede hochwertige wissenschaftliche Theorie den klärenden Rahmen $\mathrm{zu}$ einem gegebenen Forschungsgegenstand und ermöglicht sinngebende, originelle sowie leistungsfähige Vorhersagen. Aus diesen Vorhersagen können schließlich Hypothesen formuliert und anhand gezielter empirischer Beobachtung belegt werden. Mitunter liefern Vorhersagen aus Einsteins Arbeiten zur Gravitation auch nach mehr als hundert Jahren noch Raum für produktive und bestätigende Nachweise im Kontext sich sukzessive verfeinernder technologischer Möglichkeiten (vgl. bspw. Abuter et al. 2018). Mit anderen Theorien - einschließlich der für den Gegenstandsbereich der vorliegenden Arbeit relevanten Evolutionstheorie - verhält es sich ebenso: Sie stellen einen umfangreichen und nicht nur konsistenten, sondern Mehrwert bringenden, kohärenten Erklärungshintergrund für eine große Zahl empirischer Phänomene sowie etablierter Fakten und ermöglichen darüber hinaus leistungsfähige Voraussagen $\mathrm{zu}$ bisher noch nicht formulierten oder untersuchten Hypothesen. Die Leistungsfähigkeit der abgeleiteten Vorhersagen definiert mitsamt der Erklärungsleistung gegenüber bereits etablierten empirischen Phänomenen den Wert einer Theorie, während einzelne Komponenten von Theorien bzw. Vorhersagen von Theorien in Form von Hypothesen durch Herangehensweisen im Sinne der Prinzipien der Verifikation und der Falsifikation stringent getestet werden können. 


\subsection{Limitationen dieser Prinzipien und die Begründung der Metawissenschaft}

Doch auch durch eine gewissenhafte Forschung im Kontext dieser Prinzipien mitsamt der Wahl adäquater Studiendesigns, Methoden und Versuchsprotokolle ist eine Fehlerfreiheit oder auch nur eine ausreichende Treffsicherheit der Ergebnisse nicht automatisch gegeben. Eine Wirklichkeit, die ihre Regeln und Abläufe nicht transparent offenbart, durch wissenschaftliche Vorgehensweisen $\mathrm{zu}$ penetrieren, gestaltet sich schwieriger als innerhalb der Vorstellung von Laien gemeinhin angenommen wird. Faktoren wie Störvariablen, Scheinkausalität aufgrund undurchsichtiger Korrelationen, statistische Fluktuationen, Unvollkommenheit wissenschaftlicher Methoden sowie kognitive Verzerrungen seitens der Forschergemeinde begründen und schüren Zweifel an Ergebnissen wissenschaftlicher Untersuchungen. Eine hieraus abgeleitete prinzipielle Ablehnung des wissenschaftlichen Prozesses wäre freilich töricht und hätte verheerende Folgen für ein weiteres Streben nach potenziellen Fortschritten jenseits dessen, das innerhalb der bisherigen Wissenschaftsgeschichte erreicht wurde. Die Menschheit betrat den Mond nicht zufällig und erhöhte die Lebenserwartung um mehr als das Doppelte nicht planlos, sondern dank den fortwährenden, gewissenhaften und erfolgreichen Bemühungen einer Vielzahl von Wissenschaftlern. Das wissenschaftliche Programm als Ganzes demonstriert seinen Erfolg durch jene und unzählige weitere Errungenschaften. Anstatt also einen unangemessen übersteigerten Skeptizismus aus jenen problematischen Faktoren zu schlussfolgern, gilt es, die Fehleranfälligkeit der wissenschaftlichen Methoden um ein größtmögliches Maß zu reduzieren. Die nachfolgenden Beschreibungen skizzieren einen Teil ebendieses Programms.

Eine Gruppe vergleichsweise junger Vorgehensweisen bzw. Studientypen wie die Metastudie, die Metaanalyse und die systematische Übersichtsarbeit kann unter dem Deckmantel der Metawissenschaft zusammengefasst werden und soll im Nachfolgenden in ebendieser Weise benannt werden. Gemeint ist hierbei freilich nicht die philosophische Disziplin der Wissenschaftstheorie in einem engen Sinn, in der die Grundlagen wissenschaftlicher Erkenntnis und wissenschaftlichen Vorgehens selbst Gegenstand von Überlegungen und Untersuchungen sind, auch wenn Berührungspunkte sicherlich bestehen. Stattdessen sei mit diesem Begriff die von der primären Forschung abgelöste, hierarchisch übergeordnete Betrachtung einer gegebenen Forschungsfrage oder Forschungslandschaft $\mathrm{zu}$ bezeichnen. Während also im regulären, primären wissenschaftlichen Prozess konkrete Studien bzw. Untersuchungen durchgeführt werden, so betrachten metawissenschaftliche Herangehensweisen die 
bereits bestehenden Veröffentlichungen aus jener vom primären Forschungskontext abgelösten Perspektive und schaffen durch ihre eigenen Methoden einen übergreifenden Gesamtkontext zwischen ebendiesen Vorarbeiten. Auf diese Weise können vereinzelte fehlerbehaftete Studien durch die Synthese des erweiterten Forschungskontextes relativiert werden und mitunter entsteht überdies die Möglichkeit, aus einer auf den ersten Blick chaotischen Forschungslandschaft $\mathrm{zu}$ stark umstrittenen Forschungsfragen adäquate Urteile zutage $\mathrm{zu}$ fördern.

Ein konkretes Beispiel soll dieses Vorgehen weiter verdeutlichen: Die englischsprachige Meta-Datenbank PubMed, welche ihren Fokus auf den Bereichen der Medizin, Biologie, Psychologie und daran angeschlossener Disziplinen hat, katalogisierte im Mai $2019^{3}$ bereits mehr als 20.000 Studien zur Akupunktur in alleiniger oder komplementärer Anwendung. Ziel dieses außerordentlichen Forschungsvolumens ist freilich die Feststellung der Wirksamkeit dieser große Popularität genießenden Behandlungsmethode, wobei sich eine nennenswerte Problematik in der Bewertung jener Studienlage dadurch ergibt, dass sich innerhalb der Veröffentlichungen parallel jeweils viele tausend Studien mit positivem, negativem und neutralem Ergebnis auffinden. Mithin ist einem jeden einschlägig interessierten Laien, Amateur oder auch Wissenschaftler die Möglichkeit gegeben, die jeweils eigene intuitive Meinung in quasi beliebigem Umfang zu belegen und damit scheinbar zu legitimieren. Eine adäquate Beantwortung der Frage, ob Akupunktur jedoch tatsächlich wirksam ist, verschwimmt in Anbetracht der nicht überblickbaren Masse der einschlägigen Literatur mit ihren diametral entgegengesetzten Ergebnissen.

\subsection{Methodische Ansätze und Studiendesigns von Metastudien}

Trotz dieser scheinbar hoffnungslosen Situation der primären Forschung erschließen sich leistungsfähige Aussagen zum Forschungsgegenstand im Kontext metawissenschaftlicher Methoden. Eine erste notwendige Klärung besteht in der Anmerkung, dass nicht jede Studie den gleichen Wert aufweist. Ein Exkurs in die Qualitätskriterien empirischer Studien sprengt den hier gegebenen Rahmen, jedoch kann ohne besonderen Aufwand darauf hingewiesen werden, weshalb eine placebokontrollierte, randomisierte, doppelblinde, statistisch signifikante und mit einer bedeutenden Effektgröße versehene experimentelle

3 Abruf über PubMed https://pubmed.ncbi.nlm.nih.gov/ am 10. Mai 2019, 16:07 Uhr. 
Studie einer einfachen und offenen Fallstudie, bei der ein angeblicher Effekt beobachtet werden konnte, methodisch überlegen ist: Letztere leidet unter einer Reihe von methodisch nicht kontrollierten Risiken, welche die Validität der Studie untergraben und dazu führen, dass etwaige Schlussfolgerungen gerechtfertigterweise harschem Zweifel auszusetzen sind.

Hier ergibt sich ein Ansatzpunkt für metawissenschaftliche Auswertungen. Ein erster Prototyp der Metaanalyse sucht, statistische Begrenzungen kleinerer Studien zu beheben, indem die Datensätze mehrerer Studien zusammengefasst und gemeinsam ausgewertet werden. Der hiermit hergestellte, gesammelte Datensatz ist als statistisch robuster und aussagekräftiger zu sehen als die jeweiligen kleineren Datensätze der Einzelstudien. Obwohl diese Vorgehensweise prinzipiell als wirkungsvoll anzusehen ist, haben solche Metastudien mit statistischer Basis durchaus selbst wiederum inhärente Probleme. So existiert beispielsweise eine Verzerrung hin zu positiven Daten in Form eines Datenartefakts durch die Tendenz, vornehmlich positive Ergebnisse zu veröffentlichen und negative Ergebnisse als das weniger befriedigende bzw. weniger ansprechende Resultat zu ignorieren - eine Verzerrung, die sowohl seitens der Forscher als auch seitens der Fachzeitschriften auftritt, in der einschlägigen Literatur schon lange bekannt ist (Sterling 1959) und daher hinsichtlich potenzieller Lösungsstrategien beforscht wird (vgl. bspw. Egger et al. 1997). Auch durch eine selektive Auswahl oder durch eine bewusste respektive unbewusste Ausnutzung von Freiheitsgraden seitens der Forscher (vgl. bspw. Wicherts et al. 2016) kann eine Metaanalyse ebenso wie primäre Studien ihren Wert verlieren, wie beispielsweise anhand der umfassend kritisierten Metastudie von Vickers et al. (2012) zur Wirksamkeit von Akupunktur aufgezeigt werden kann. Dennoch sind derartige Auswertungen in manchen Forschungskontexten sowie unter der Voraussetzung, dass sie mit großem Pflichtbewusstsein eingesetzt werden, ein hilfreiches Werkzeug.

Andere Metastudien entstehen nicht auf einer derartigen statistischen Basis, sondern berücksichtigen in qualitativer Hinsicht die Arbeitsweise und die Ergebnisse der zugrundeliegenden Studien. Systematische Übersichtsarbeiten bzw. Reviews suchen, das in der Literatur verfügbare Wissen zu sammeln und zusammenfassend sowie bewertend wiederzugeben. Ein damit verbundenes Ziel ist die Aufbereitung der jeweiligen Forschungslandschaft für Personen, die sich nicht aktiv innerhalb der einschlägigen Forschung aufhalten, aber vom dort fixierten Wissen profitieren können, womit derartigen Metastudien eine gewichtige Rolle zuzusprechen ist. Obwohl auch hier ein wichtiges Format wissenschaftlicher Erkenntnis gegeben ist und derartige Veröffentlichungen weithin ihren Zweck adäquat erfüllen, so scheint ein derartiges Vorgehen wiederum 
von der fachlichen und methodischen Kompetenz des Autors bzw. der Autoren abhängig zu sein und außerdem potenziell Objekt eines Einflusses aufgrund persönlicher Neigungen zu sein. In der Tat ist sicherlich der Fall, dass derartige als problematisch anzusehende Reviews durchaus existieren (vgl. bspw. nochmals Vickers et al. 2012 oder auch die inzwischen zurückgezogene Studie von Zhu, Ma \& Deng 2018), jedoch fand die einschlägige Literatur im Streben nach qualitativer Selbstkontrolle zu methodischen Ansätzen, um hochwertige Metastudien von minderwertigen zu unterscheiden.

Vormals wurde bereits erwähnt, dass primäre Studien Qualitätskriterien aufweisen oder missen können. Leistungsfähig konstruierte Metastudien nehmen diesen Umstand in ihre Analyse auf und untersuchen die Grundgesamtheit oder eine Teilmenge der verfügbaren Studien zu einer gegebenen Forschungsfrage in einem Vorgehen, welches die Ergebnisse einer Studie mit ihrer Qualität zu korrelieren sucht. So zeigt sich am Beispiel der Wirksamkeit von Akupunktur, dass innerhalb der einschlägigen Literatur Studien mit positivem, negativem und neutralem Ergebnis im Rahmen einer ungefilterten Recherche parallel existieren, aber dass dieses Urteil einer Parallelität bei einer Filterung nach Studienqualität nicht umfänglich aufrechterhalten werden kann. Im Bereich niedriger Qualität spiegelt sich dieses Bild wider, jedoch zeigen die qualitativ hochwertigsten Studien eine überwältigende Tendenz zur Verneinung jeglicher Wirksamkeit - und ihrerseits hochwertige und pflichtbewusst durchgeführte Metastudien kommen zum selben Ergebnis (vgl. für eine methodisch stringente und Teilfragen triangulierende Primärstudie Cherkin et al. 2009 sowie für metawissenschaftliche Kommentare und Reviews Ernst, Lee \& Choi 2011, Hall 2011 sowie Colquhoun \& Novella 2013).

In der Tat beschäftigt sich ein eigener Zweig der Forschung mit der effizienten Durchführbarkeit von möglichst aussagekräftigen Metastudien und brachte in der jüngeren Forschung Ansätze wie Slavins (1989 und 1995) „best evidence synthesis“ hervor, welche hinsichtlich ihrer Anwendbarkeit weiterhin untersucht und verfeinert wird (vgl. bspw. Treadwell et al. 2012). Laut der dort erarbeiteten Schlussfolgerungen reicht die Rezeption einer vergleichsweise kleinen Auswahl der besten verfügbaren Studien aus, um das selbe Ergebnis wie eine erschöpfende Literaturrezeption zu erreichen, womit sich die Effizienz metawissenschaftlichen Vorgehens nennenswert erhöht.

Zuletzt ist darauf hinzuweisen, dass verschiedene Studientypen wie klassische Metaanalysen und systematische Übersichtsarbeiten als sich gegenseitig ergänzend in einer einzigen Veröffentlichung durchgeführt werden können. Das Feld der Metastudien zeigt sich damit wandelbar und flexibel sowie als sich zunehmend methodisch verfeinernd. Eine Reihe von quantitativen und qualita- 
tiven Herangehensweisen erlauben dabei höchst leistungsfähige Einschätzungen zur jeweils einschlägigen Datenlage und mitunter sind in manchen Forschungskontexten allein durch hochwertige Metastudien abschließende Antworten zu erreichen. In diesem Sinne läuft ein jedes Forschungsfeld, das sich noch nicht metawissenschaftlich mit seiner eigenen Forschungslandschaft auseinandersetzt, als Ganzes Gefahr, sich von zuvor erwähnten Limitationen der wissenschaftlichen Methodologie nicht emanzipieren zu können. Die vorliegende Arbeit sieht sich - wie zuvor angemerkt wurde und nachfolgend skizziert werden soll - als Agent einer solchen metawissenschaftlich begründeten Mündigkeit mit Hinblick auf die Sprachursprungsforschung. Bevor zu weiteren Erläuterungen fortgeschritten werden soll, sei noch knapp auf Littell, Corcoran \& Pillai (2008) als eine allgemeine Einführung zum Feld der Metastudien zu verweisen.

\subsection{Interdisziplinarität als eine besondere Herausforderung}

Wie im Bisherigen dargestellt steigern klassische sowie jüngere, komplementäre metawissenschaftliche Ansätze die Kraft des wissenschaftlichen Vorgehens, indem innerhalb deren methodischer Programme Forschungslandschaften synthetisiert und geschlossen ausgewertet werden können. Jenseits dieser „internen“ Analyse im Sinne des Diskutierten stellt sich jedoch sowohl hinsichtlich des Gegenstands der vorliegenden Arbeit als auch im Kontext anderer Forschungsfelder ein weiteres Problem, welches als äußerst schwerwiegend einzuschätzen ist. All jene wissenschaftlichen Prinzipien und Vorgehensweisen mitsamt den erläuterten metawissenschaftlichen Strategien bewegen sich prototypisch innerhalb einer konkreten Disziplin und suchen dort nach größtmöglicher Leistungsfähigkeit. Ein explizit interdisziplinärer Forschungsgegenstand führt jedoch in geradezu systematischer Weise zur schwerwiegenden Problematik einer erzwungenen Kooperation bei gegenseitigem Nichtverstehen.

Hierzu ist zunächst anzumerken, dass Forscher freilich prinzipiell in der Lage sind, sich außerhalb ihrer eigenen Expertise zu bewegen und ein adäquates fachfremdes Verständnis aufzubauen, jedoch handelt es sich dabei um ein umfassend mit Schwierigkeiten versehenes Unterfangen. Fachfremd erworbene Perspektiven laufen Gefahr, unvollständig, fehlerhaft oder unscharf zu verbleiben - und derartige blinde Flecken bzw. tote Winkel führen potenziell zu schwerwiegenden Fehleinschätzungen bzw. zu problematischen Missverständnissen. Ein interdisziplinärer Forschungsgegenstand stellt damit besondere Herausforderungen, welche selbst durch die bereits vorgestellten metawissenschaftlichen Lösungsstrategien nicht problemlos bewältigt werden können. 
Diese interdisziplinären Schwierigkeiten begründen sich in mehreren Umständen: Erstens fußen sie darin, dass nicht alle wissenschaftlichen respektive metawissenschaftlichen Vorgehensweisen zu jedem Forschungsfeld passen, denn die Natur des jeweiligen Forschungsfeldes bestimmt die (meta)wissenschaftlichen methodischen Möglichkeiten. Daraus ergibt sich auf beinahe triviale Weise eine wenigstens teilweise Unvergleichbarkeit und Unvereinbarkeit der Methoden, womit einerseits beide Parteien intuitiv skeptisch auf die jeweils andere blicken und andererseits keine direkte metawissenschaftliche Synthese im Sinne des bisher Skizzierten möglich ist.

Zweitens beginnen Differenzen oftmals schon bei der Operationalisierung von Begrifflichkeiten und der Perspektive auf den Forschungsgegenstand. Selbst augenscheinlich sehr nahe Disziplinen wie die Physik und die Chemie, welche hinsichtlich der Erforschung von Elementarteilchen, Atomen und Molekülen überlappen und populär als intuitiv vergleichbare Naturwissenschaften verstanden werden, unterscheiden sich in ihrer Herangehensweise, ihrer Perspektive und ihren Methoden derart, sodass sie sich bis heute als jeweils eigene Arbeitsfelder behaupten konnten und sodass es einem eigenen Arbeitsbereich dem der physikalischen Chemie - bedarf, um hinsichtlich konkreter Fragestellungen eine interdisziplinäre Arbeit produktiv zu gestalten. Ähnliches gilt an Berührungspunkten jener beiden Disziplinen mit der Biologie, sodass sich im Verlauf der jüngeren Wissenschaftsgeschichte auch die Biochemie, die Biophysik sowie die biophysikalische Chemie zu äußerst umfangreichen und leistungsfähigen Forschungsbereichen entwickeln konnten. Nun sind diese Entwicklungen als durchaus erfolgreich und produktiv zu sehen, jedoch unterstreichen sie trotz dieses Umstands die generelle Problematik der Interdisziplinarität - und sobald Psychologen, Neurowissenschaftler und Philosophen eine Diskussion zum menschlichen Bewusstsein beginnen, zeigt sich die dunkle Seite der Interdisziplinarität in vollem Umfang.

Drittens besteht beim Austausch zwischen Experten unterschiedlicher Disziplinen das selbe Problem wie beim Austausch eines Experten mit einem Laien: Die Ebene des Diskurses, auf dem sich die Diskutanten bewegen, ist voneinander in einer Weise abgehoben, sodass beide Seiten für die jeweils gegenüberliegende Partei schlicht ein Mysterium darstellen. Wie ein jeder Experte, der jemals suchte, einen Laien zur eigenen Forschung aufzuklären, bezeugen kann, erscheint eine derartige Situation geradezu hoffnungslos, da der jeweilige Laie viele Jahre strenger einschlägiger Ausbildung und Bildung vermisst - Jahre des fachlichen und methodischen Trainings, welche den Diskurs des Experten fundieren. Eine typische Folge des Aufstiegs zum Experten besteht darin, dass eine Einsicht in die mentale Welt des Laien verloren geht. Wissen, Perspektive und 
Terminologie werden seitens des Experten als selbstverständlich angenommen. Zugleich kann ein Laie den fachlichen, methodischen und perspektivischen Weg, den ein Experte auf dem Weg zum Expertentum zurückgelegt hat, kaum ermessen. Experten verschiedener Disziplinen sind zueinander wenigstens näherungsweise als Laien zu sehen, womit auch in dieser Hinsicht die interdisziplinäre Problematik geschürt wird.

Die Folge aus alledem ist freilich, dass an Berührungspunkten zwischen wenig vereinbaren (Teil-)Disziplinen Konflikte entstehen und dass zwei derartige Forschungslandschaften nicht in einer Weise synthetisiert werden können, wie es innerhalb einer einzelnen Disziplin möglich ist. Dennoch besteht durch metawissenschaftliche Prinzipien und Vorgehensweisen ein mächtiges Instrument, mit dem auch in interdisziplinären Forschungsfeldern ein nennenswerter analytischer Mehrwert erreicht werden kann. Die vorliegende Arbeit sucht dies $\mathrm{zu}$ demonstrieren, indem mehrere qualitative Vorgehensweisen - sowohl bereits vorgestellte als auch bisher unerwähnt verbliebene - zu einem Methodenkatalog vereint werden sollen. Dieser an späterer Stelle vorzustellende Methodenkatalog stellt ein informierendes Instrument dar, um die zerklüftete interdisziplinäre Forschungslandschaft zum Ursprung menschlicher Sprachbefähigung stringenter zusammenzufassen und aufzubereiten als gemeinhin innerhalb der einschlägigen Literatur geschehen ist. Eine exemplarische Anwendung der erarbeiteten Methodologie soll die Leistungsfähigkeit dieses Ansatzes weiter unterstreichen und der vorliegenden Arbeit zusätzlichen Mehrwert verschaffen. All dem voranzustellen sind jedoch die nachfolgenden Kapitel, welche zusätzlich zu den vorliegenden methodischen Fundamenten einige fachliche Verständnisgrundlagen skizzieren. 